\title{
Phases of the electronic two-level model under the rotating wave approximation
}

\author{
M.T. Thomaz ${ }^{1 *}$ A.C. Aguiar Pinto ${ }^{2}$ and M. Moutinho ${ }^{2}$ \\ ${ }^{1}$ Instituto de Física, Universidade Federal Fluminense, \\ Av. Gal. Milton Tavares de Souza s/no, CEP 24210-346, Niterói-RJ, Brazil \\ ${ }^{2}$ Curso de Física, Universidade Estadual de Mato Grosso do Sul, \\ Caixa Postal: 351, Cidade Universitária de Dourados, CEP 79804-970, Dourados-MS, Brazil.
}

November 16, 2018

\begin{abstract}
We present the time evolution of the electronic two-level model in the rotating wave approximation (RWA). We calculate the Aharonov-Anandan phase of the vector states with cyclic evolution either within the period of an external monochromatic electric field or the period corresponding to Rabi's frequency. The AharonovAnandan phase is shown to be dependent on the initial vector state, unless the system evolves in the adiabatic regime; in the latter case, the Aharonov-Anandan phase recovers Berry's phase. Our results are also discussed in the quasi-resonant regime.
\end{abstract}

Keywords: Two-level model, Aharonov-Anadan phases, electric dipole approximation, rotating wave wave approximation (RWA)

PACS numbers: 03.65.Vf, 03.65.-w, 03.65.Aa

\footnotetext{
*Corresponding author: mtt@if.uff.br
} 


\section{Introduction}

A quantum system coupled to a classical monochromatic laser in the quasi-resonant regime can be approximated by a $N$-level model, $N$ being finite, in the rotating wave approximation (RWA) [1].

The RWA of the two-level model has been used as a candidate for modeling geometric phase gates between ion-qubits in the presence of an external electric or magnetic field[2]. These two-level models can be realized through the interaction of an external magnetic field with a spin-1/2[3, 4], or matter coupled to an external electric field, in the dipole approximation[1].

Recently we have studied the adiabatic evolution of a two-level model[5] $(i)$ under a (real) classical monochromatic electric field and ( $i i)$ when only the contribution of the positive frequency of the electric field is taken into account (its RWA). In the RWA of the two-level model we recover the geometric phases acquired by the instantaneous eigenstates of energy already known in the literature [6]. On the other hand, we verified the absence of geometric phase in the adiabatic evolution of the instantaneous eigenstates of the two-level model coupled to a real monochromatic electric field.

Let $\omega$ be the angular frequency of the monochromatic external electric field and $\Delta \varepsilon$ the energy difference of the two-level model. The matter-field coupling can only be approximated by two-level model in the quasi-resonant regime 1 , in which $\omega \approx \Delta \varepsilon$ (in natural units, $c=\hbar=1$ ). Certainly this condition does not fulfill the adiabatic condition[5] $\omega \ll \Delta \varepsilon$. In 2002, Xin et al. [7] proposed a scheme to perform quantum geometric computation in non-adiabatic regime with trapped ions.

Recently Imai et al. [8] and Imai and Morinaga [9] measured the geometric phases in the two-level model coupled to a monochromatic electric field. In Refs. [8, 9] it is mentioned that "A geometric phase solely depends on the amount of the solid angle enclosed by the evolution path, not on the details ..., or the initial and final states of the evolution.". This statement is certainly true when the quantum model is under adiabatic evolution[10], but it is not obvious that this comment is valid in the RWA of the two-level model coupled to a monochromatic electric field in the quasi-resonant regime $\left(\omega=\Delta \varepsilon+\delta\right.$, and $\left.\frac{\delta}{\Delta \varepsilon} \ll 1\right)$.

The present work aims at studying the dynamics the two-level model under the RWA and coupled to a linearly polarized monochromatic electric field, in the electric dipole approximation [1], for any value of the ratio $\frac{\omega}{\Delta \varepsilon}$. We want to determine the initial conditions under which a vector state acquires an Aharonov-Anandan phase[11] within different periods. Our model is simpler than the one studied in Refs. [8, 9], where different laser beams are applied to matter in order that the quantum system acquire the geometric phases.

In section 2 we present the hamiltonian of model under study. Its dynamics is derived by mapping it onto a spin-1/2 model coupled to an effective magnetic field. The study of global phases in the non-adiabatic evolution of the spin-1/2 model in the presence of a rotating magnetic field was carried out previously in Refs. [12, 13, 14]. In section 3 we study the cyclic evolution of the vector states under the action of the electric twolevel model. The Aharonov-Anandan phase acquired by the states are considered for state vector with periodicity within that of the external electric field or that of Rabi's frequency. Our results can be used to extend easily the discussions made in Refs. [12, 13, 14, on these 
phases in the spin-1/2 model under the action of a precessing magnetic field. A summary of our results is presented in section 4 . Appendix A presents the average energy in any vector state.

\section{The dynamics of the RWA of the two-level model}

We begin with the hamiltonian $\mathbf{H}_{0}$ of the quantum two-level model, without interaction[15],

$$
\mathbf{H}_{0}=\varepsilon_{1}|1\rangle\left\langle 1\left|+\varepsilon_{2}\right| 2\right\rangle\langle 2|,
$$

where $\mathbf{H}_{0}|i\rangle=\varepsilon_{i}|i\rangle, i=1,2$. We take $\varepsilon_{1}<\varepsilon_{2}$, which prevents (11) from describing a degenerate two-level system. We use the natural units: $c=\hbar=1$.

The two-level interaction hamiltonian of the matter and the electric field, in the electric dipole approximation, $\mathbf{H}_{e}(t)$, represented in the basis of the eigenstates of $\mathbf{H}_{0}$, is [16]

$$
\mathbf{H}_{e}(t)=\left(\begin{array}{cc}
\varepsilon_{1} & \vec{d}_{12} \cdot \vec{E}(t) \\
\left(\vec{d}_{12} \cdot \vec{E}(t)\right)^{*} & \varepsilon_{2}
\end{array}\right)
$$

in which

$$
\vec{d}_{12} \equiv-e\langle 1|\overrightarrow{\mathbf{x}}| 2\rangle
$$

and $\overrightarrow{\mathbf{x}}$ is the position operator of the electron. We assume that the eletronic states $|i\rangle$, $i=1,2$, are spherically symmetric, and hence do not have a permanent electric dipole $\left(\vec{d}_{i i}=0, i=1,2\right)$. Throughout our calculations, we have chosen the basis of eigenstates of $\mathbf{H}_{0}$, such that the components of $\vec{d}_{12}$ are real; we have taken $e>0$, so that the electronic charge is $-e$.

In the RWA, the main contribution to the quantum phenomenon comes from the positive angular frequency of the monochromatic electric field. In this approximation the classical electric field in hamiltonian (2) becomes

$$
\vec{E}(t) \approx \vec{E}_{0} e^{-i\left(\omega t-\phi_{0}\right)},
$$

where $\vec{E}_{0} \in \mathbb{R}^{3}, \omega>0$ and the phase $\phi_{0}$ is chosen such that $\vec{d}_{12} \cdot \vec{E}_{0} \geq 0$. For the complex electric field (4), the hamiltonian (2) depends on the two dimensional variable $\left(\vec{d}_{12} \cdot \vec{E}(t)\right)$.

The dynamics of the spin- $1 / 2$ coupled to a magnetic field that precess around a fixed direction has been studied previously for any dynamical regime[17, 18].

By making a shift in hamiltonian (2), it can be written as the hamiltonian of a spin- $1 / 2$ coupled to an effective magnetic field, that is,

$$
\mathbf{H}_{e}(t)=\frac{\left(\varepsilon_{1}+\varepsilon_{2}\right) \mathbf{1}}{2}+\frac{\mu}{2} \vec{B}_{e f f}(t) \cdot \vec{\sigma}
$$


where 1 is the identity operator of dimension $2 \times 2, \vec{\sigma}$ are the Pauli matrices, $\mu=\mu_{B} g$, with $g$ being the Landé's factor and $\mu_{B}$ the Bohr magneton. The components of the effective magnetic field on the r.h.s. of eq.(5) are,

$$
\begin{aligned}
& B_{x}^{e f f}(t)=\frac{2 D_{0}}{\mu} \cos \left(\omega t-\phi_{0}\right), \\
& B_{y}^{e f f}(t)=\frac{2 D_{0}}{\mu} \sin \left(\omega t-\phi_{0}\right), \\
& B_{z}^{e f f}(t)=-\frac{\Delta \varepsilon}{\mu}
\end{aligned}
$$

where

$$
D_{0} \equiv \vec{d}_{12} \cdot \vec{E}_{0} \geq 0
$$

and

$$
\Delta \varepsilon \equiv \varepsilon_{2}-\varepsilon_{1}>0
$$

The eigenvectors of hamiltonian $\mathbf{H}_{0}(|i\rangle, i=1,2)$ are also eigenvectors of the operator $\sigma_{z}$, that is, $\sigma_{z}|1\rangle=|1\rangle$ and $\sigma_{z}|2\rangle=-|2\rangle$.

The components of the effective magnetic field $\vec{B}_{\text {eff }}(t)$ can also be written in terms of polar coordinates, $\vec{B}_{e f f}(t)=\left(B \sin (\theta) \cos \left(\omega t^{\prime}\right), B \sin (\theta) \sin \left(\omega t^{\prime}\right), B \cos (\theta)\right)$, where $t^{\prime} \equiv$ $t-\frac{\phi_{0}}{\omega}$ and

$$
\begin{aligned}
& \cos (\theta)=-\frac{1}{2} \frac{\Delta \varepsilon}{\sqrt{D_{0}^{2}+\left(\frac{\Delta \varepsilon}{2}\right)^{2}}} \\
& \sin (\theta)=\frac{D_{0}}{\sqrt{D_{0}^{2}+\left(\frac{\Delta \varepsilon}{2}\right)^{2}}} .
\end{aligned}
$$

The norm $B$ of the effective magnetic field is

$$
B=\frac{2}{\mu} \sqrt{D_{0}^{2}+\left(\frac{\Delta \varepsilon}{2}\right)^{2}}
$$

The effective magnetic field on the r.h.s. of hamiltonian (5) has constant norm and constant $z$-component. It precesses around the $z$ direction with angular velocity $\omega$. From expressions (8a $)$ and (8b), we conclude that $\theta \in\left[\frac{\pi}{2}, \pi\right]$.

The eigenvectors and the eigenvalues of the second term on the r.h.s. of hamiltonian (5) were calculated in Refs.[12, 13, 17, 18]. The eigenvectors of the hamiltonian of a spin- $1 / 2$ coupled to a precessing magnetic field calculated in these two references differ by global phases. From now on, we follow Ref.[17. 
The eigenvalue equation of $\mathbf{H}_{e}(t)$ is

$$
\mathbf{H}_{e}(t)\left|\phi_{i} ; i\right\rangle=E_{i}\left|\phi_{i} ; i\right\rangle, \quad i=1,2
$$

where

$$
E_{i}=\frac{\left(\varepsilon_{1}+\varepsilon_{2}\right)}{2}+\tilde{E}_{i}, \quad i=1,2,
$$

with

$$
\tilde{E}_{1}=-\sqrt{D_{0}^{2}+\left(\frac{\Delta \varepsilon}{2}\right)^{2}} \quad \text { and } \quad \tilde{E}_{2}=\sqrt{D_{0}^{2}+\left(\frac{\Delta \varepsilon}{2}\right)^{2}} .
$$

The eigenvectors of $\mathbf{H}_{e}(t)$ associated to $E_{1}(t)$ and $E_{2}(t)$ are respectively:

$$
\begin{aligned}
\left|\phi_{1} ; t\right\rangle & =-\sin \left(\frac{\theta}{2}\right)|1\rangle+\cos \left(\frac{\theta}{2}\right) e^{i\left(\omega t-\phi_{0}\right)}|2\rangle, \\
\left|\phi_{2} ; t\right\rangle & =\cos \left(\frac{\theta}{2}\right)|1\rangle+\sin \left(\frac{\theta}{2}\right) e^{i\left(\omega t-\phi_{0}\right)}|2\rangle .
\end{aligned}
$$

From eqs. (8a and (8b) we obtain

$$
\begin{aligned}
& \cos \left(\frac{\theta}{2}\right)=\frac{1}{\sqrt{2}}\left[1-\frac{\left(\frac{\Delta \varepsilon}{2}\right)}{\sqrt{D_{0}^{2}+\left(\frac{\Delta \varepsilon}{2}\right)^{2}}}\right]^{1 / 2}, \\
& \sin \left(\frac{\theta}{2}\right)=\frac{1}{\sqrt{2}}\left[1+\frac{\left(\frac{\Delta \varepsilon}{2}\right)}{\sqrt{D_{0}^{2}+\left(\frac{\Delta \varepsilon}{2}\right)^{2}}}\right]^{1 / 2} .
\end{aligned}
$$

From the previous expressions of the eigenvalues and of the eigenvectors of $\mathbf{H}_{e}(t)$, we verify that the ratio $\left(\frac{2 D_{0}}{\Delta \varepsilon}\right)$ characterizes the coupling strength between the matter and the external electric field: i) $\frac{2 D_{0}}{\Delta \varepsilon} \ll 1$, means a weak coupling; ii) $\frac{2 D_{0}}{\Delta \varepsilon} \gg 1$, means to a strong coupling. We remind that the strength of the coupling depends not only upon the norm of the electric field but also on its relative direction to the vector $\vec{d}_{12}$ (see eq.(7a $)$ ).

Let us consider the initial vector state $|\psi(0)\rangle$,

$$
|\psi(0)\rangle=c_{1}(0)\left|\phi_{1} ; 0\right\rangle+c_{2}(0)\left|\phi_{2} ; 0\right\rangle
$$

where $c_{1}(0)$ and $c_{2}(0) \in \mathbb{C}$. The states $\left|\phi_{i} ; 0\right\rangle, i=1,2$, are equal to the states eqs.(12a) and (12b) at $t=0$. These coefficients satisfy the normalization condition: $\left|c_{1}(0)\right|^{2}+\left|c_{2}(0)\right|^{2}=$ 1. 
The time evolution of the vector $|\psi(t)\rangle$ is

$$
\mathbf{H}_{e}(t)|\psi(t)\rangle=i \frac{d|\psi(t)\rangle}{d t}
$$

and it is subjected to the initial condition (14).

Using the instantaneous eigenstates of energy (12a) and (12b) to decompose the vector $|\psi(t)\rangle$,

$$
|\psi(t)\rangle=\sum_{j=1}^{2} c_{j}(t) e^{-i E_{j} t}\left|\phi_{j} ; t\right\rangle,
$$

and substituting eq.(16) in the Schrödinger equation (15), we obtain the equations satisfied by the coefficients $c_{1}(t)$ and $c_{2}(t)$. These equations are identical to eqs. (12) and (13) of Ref.[17] if in the latter equations we make the following replacements: $\omega_{1}=\frac{E_{2}-E_{1}}{2}$ and $\omega_{0}=\omega$.

To simplest expressions of the coefficients, we include the dynamical phases in them and define

$$
\tilde{c}_{j}(t) \equiv c_{j}(t) e^{-i E_{j} t}, \quad j=1,2 .
$$

The expressions of the coefficients $\tilde{c}_{j}(t), j=1,2$, at any instant $t$, are

$$
\begin{gathered}
\tilde{c}_{1}(t)=e^{-\frac{i}{2}\left(\varepsilon_{1}+\varepsilon_{2}+\omega\right) t}\left\{c_{1}(0) \cos (\Gamma t)+i \frac{\sin (\Gamma t)}{\Gamma}\left[\left(\tilde{E}_{2}-\frac{\omega}{2} \cos (\theta)\right) c_{1}(0)\right.\right. \\
\left.\left.-\frac{\omega}{2} \sin (\theta) c_{2}(0)\right]\right\} \\
\tilde{c}_{2}(t)=e^{-\frac{i}{2}\left(\varepsilon_{1}+\varepsilon_{2}+\omega\right) t}\left\{c_{2}(0) \cos (\Gamma t)-i \frac{\sin (\Gamma t)}{\Gamma}\left[\left(\tilde{E}_{2}-\frac{\omega}{2} \cos (\theta)\right) c_{2}(0)\right.\right. \\
\left.\left.+\frac{\omega}{2} \sin (\theta) c_{1}(0)\right]\right\},
\end{gathered}
$$

where $\Gamma$ is the Rabi's frequency and

$$
\begin{aligned}
\Gamma & =\frac{1}{2} \sqrt{(\mu B-\omega \cos (\theta))^{2}+\omega^{2} \sin ^{2}(\theta)} \\
& =\sqrt{D_{0}^{2}+\frac{1}{4}(\Delta \varepsilon+\omega)^{2}} .
\end{aligned}
$$

The expressions (18a) and (18b) are valid for any value of the ratio $\left(\frac{\omega}{\Delta \varepsilon}\right)$, including the quasi-resonant regime. 


\section{The Aharonov-Anandan phase}

In 1987 Aharonov and Anandan extended the concept of geometric phase to any time evolution of a quantum system[11]. Suppose the vector state $|\psi(t)\rangle$ evolves under the action of an hamiltonian $\mathbf{H}(t)$. The cyclic evolution of the quantum system is related to its vector state during the period $\tau$, and

$$
|\psi(\tau)\rangle=e^{i \phi}|\psi(0)\rangle
$$

In Ref.[1], the authors define a shifted vector state $|\tilde{\psi}(t)\rangle$,

$$
|\tilde{\psi}(t)\rangle \equiv e^{-i f(t)}|\psi(t)\rangle
$$

where the function $f(t)$ satisfies the condition: $f(\tau)-f(0)=\phi$.

It is shown in this reference that

$$
\phi=\beta-\int_{0}^{\tau}\langle\psi(t)|\mathbf{H}(t)| \psi(t)\rangle d t
$$

The expression of the Aharonov-Anandan phase, the $\beta$ phase, is

$$
\beta=\int_{0}^{\tau}\langle\tilde{\psi}(t)| i \frac{d}{d t}(|\tilde{\psi}(t)\rangle)
$$

that is formally identical to the phase acquired by the instantaneous eigenstates of energy when they evolve adiabatically[10]. We point out that differently from the Berry's phase, the vector state in eq.(20) is any state that satisfies this cyclic condition.

Any initial vector state can be written as eq.(14) and at any instant $t \geq 0$ it becomes

$$
|\psi(t)\rangle=\sum_{j=1}^{2} \tilde{c}_{j}(t)\left|\phi_{j} ; t\right\rangle .
$$

The expressions of the coefficients $\tilde{c}_{1}(t)$ and $\tilde{c}_{2}(t)$ are given by eqs. (18a) and (18b) respectively, and $\left|\phi_{j} ; t\right\rangle, j=1,2$, are the instantaneous eigenstates of $\mathbf{H}_{e}(t)$ (see eqs.(12a) and $(12 \mathrm{~b})$ ). The coefficients $\tilde{c}_{j}(t), j=1,2$, satisfy the normalization condition: $\left|\tilde{c}_{1}(t)\right|^{2}+$ $\left|\tilde{c}_{2}(t)\right|^{2}=1$.

Along an adiabatic evolution, the coefficient $\tilde{c}_{j}(t), j=1,2$, differs from its respective initial coefficient $c_{j}(0), j=1,2$, by at most a phase. That is not the general case of eqs. (18a) and (18b), where for arbitrary instant $t$ we have: $\left|\tilde{c}_{j}(t)\right| \neq\left|c_{j}(0)\right|, j=1,2$.

Let us call $T$ the period of the external electric field $(\omega T=2 \pi)$. The hamiltonian (2) has also period $T, \mathbf{H}_{e}(T)=\mathbf{H}_{e}(0)$, as well as its instantaneous eigenvectors. 
We redefine the coefficients (18a) and $(\underline{18 b})$ as

$$
\begin{aligned}
& \tilde{c}_{1}(t) \equiv e^{-\frac{i}{2}\left(\varepsilon_{1}+\varepsilon_{2}+\omega\right) t} g_{1}(t), \\
& \tilde{c}_{2}(t) \equiv e^{-\frac{i}{2}\left(\varepsilon_{1}+\varepsilon_{2}+\omega\right) t} g_{2}(t) .
\end{aligned}
$$

We call $T_{\Gamma}$ the period associated to the Rabi's frequency $\Gamma\left(\Gamma T_{\Gamma}=2 \pi\right)$. From the definitions of $g_{j}(t), j=1,2$, we verify that

$$
\begin{aligned}
& g_{1}(0)=g_{1}\left(n T_{\Gamma}\right), \\
& g_{2}(0)=g_{2}\left(n T_{\Gamma}\right),
\end{aligned}
$$

where $n=0,1,2, \cdots$

\subsection{Cyclic evolution at $\tau=T$}

The vector state (16) at $\tau=T$ is

$$
|\psi(T)\rangle=-e^{-\frac{i}{2}\left(\varepsilon_{1}+\varepsilon_{2}\right) T}\left[g_{1}(T)\left|\phi_{1} ; 0\right\rangle+g_{2}(T)\left|\phi_{2} ; 0\right\rangle\right],
$$

where

$$
\begin{aligned}
& g_{1}(T)=c_{1}(0) \cos (\Gamma T)+i \frac{\sin (\Gamma T)}{\Gamma}\left[\left(\tilde{E}_{2}-\frac{\omega}{2} \cos (\theta)\right) c_{1}(0)-\frac{\omega}{2} \sin (\theta) c_{2}(0)\right] \\
& g_{2}(T)=c_{2}(0) \cos (\Gamma T)-i \frac{\sin (\Gamma T)}{\Gamma}\left[\left(\tilde{E}_{2}-\frac{\omega}{2} \cos (\theta)\right) c_{2}(0)+\frac{\omega}{2} \sin (\theta) c_{1}(0)\right] .
\end{aligned}
$$

We verify from eqs. (26) $-(27 \mathrm{~b})$ that for the vector state $|\psi(T)\rangle$ to differ by a global phase from $|\psi(0)\rangle$ (see eq.(20) ), the ratio $\left(\frac{\Gamma}{\omega}\right)$ has to have particular values or we have to choose special values for the initial coefficients $c_{1}(0)$ and $c_{2}(0)$.

We begin the discussion of $\tau=T$ with two special cases [13, 14]:

1) $\Gamma=n \omega, \quad n=1,2,3, \cdots$

For any initial vector state (14), where $c_{1}(0)$ and $c_{2}(0) \in \mathbb{C}$, the vector state at $t=T$ is written as eq.(20) with

$$
\phi=-\pi-\frac{1}{2}\left(\varepsilon_{1}+\varepsilon_{2}\right) T .
$$


To derive the Aharonov- Anandan phase defined in eq.(22a), we use eq. (A.3) to calculate the integral of the expectation value of the energy in the state $|\psi(t)\rangle$ during the interval $t \in[0, T]$. In the general case, the coefficient $c_{1}(0) \in \mathbb{C}$ and we write it as $c_{1}(0)=\left|c_{1}(0)\right| e^{i \delta_{1}}, \delta_{1} \in \mathbb{R}$.

Subtracting the dynamical phase from the global phase (28), we obtain

$$
\begin{aligned}
\beta=-\pi- & \frac{\pi}{\cos (\theta)}\left(\frac{\Delta \varepsilon}{\omega}\right)\left[\left(\left|c_{2}(0)\right|^{2}-\left|c_{1}(0)\right|^{2}\right)\left(1-\frac{\sin ^{2}(\theta)}{4 n^{2}}\right)\right. \\
& \left.+\frac{\sin (\theta)}{2 n} \sqrt{1-\frac{\sin ^{2}(\theta)}{4 n^{2}}}\left|c_{1}(0)\right|\left[c_{2}(0) e^{-i \delta_{1}}+c_{2}^{*}(0) e^{i \delta_{1}}\right]\right] .
\end{aligned}
$$

In the adiabatic limit $(\Delta \varepsilon \gg \omega)$, with $c_{2}(0)=0$, we recover from eq.(29) the Berry's phase acquired by the instantaneous eigenstate of energy $\left|\phi_{1} ; t\right\rangle$ (see eq.(26) of Ref.[17]). In the same limit, the Aharonov-Anandan phase with $c_{1}(0)=0$ is equal to Berry's phase associated to the state $\left|\phi_{2} ; t\right\rangle$ (see eq.(28) of Ref.[17]). In each case, the Berry's phase, or equivalently the Aharonov-Anandan phase in the adiabatic limit, is independent of the initial coefficients $c_{1}(0)$ and $c_{2}(0)$.

The $\beta$ phase (29) is acquired by any vector state $|\psi(t)\rangle$ at $\tau=T$ when $\Gamma=n \omega$, $n=1,2, \cdots$, but its value depends on the the initial coefficients $c_{1}(0)$ and $c_{2}(0)$, differently from what is stated in Refs. [8] and [9].

In eq. (29) we write the $\beta$ phase as a function of $\cos (\theta)$ and $\sin (\theta)$ in order to show that in the quasi-resonant regime $\left(\frac{\Delta \varepsilon}{\omega} \approx 1\right)$ this phase is not equal to the solid angle enclosed by the evolution of the effective magnetic field (6a) - (6c).

From the expression of the Rabi frequency (19b) we obtain the value of the coupling $D_{0}$ between the matter and the electric field such that the condition $\Gamma=n \omega, n=1,2, \cdots$ is satisfied

$$
D_{0}^{2}=n^{2} \omega^{2}-\frac{1}{4}(\Delta \varepsilon+\omega)^{2}, \quad n=1,2, \cdots
$$

At this point, we restrict the discussion to the quasi-resonant regime $\left(\omega=\Delta \varepsilon+\delta, \frac{\delta}{\Delta \varepsilon} \ll\right.$ 1). In this regime, eq.(30) becomes, up to first order in $\left(\frac{\delta}{\Delta \varepsilon}\right)$,

$$
\left(\frac{D_{0}}{\Delta \varepsilon}\right)^{2} \approx\left(n^{2}-1\right)+\left(2 n^{2}-1\right)\left(\frac{\delta}{\Delta \varepsilon}\right), \quad n=1,2, \cdots
$$

For $n=1$, eq.(31) is

$$
\left(\frac{D_{0}}{\Delta \varepsilon}\right)^{2} \approx \frac{\delta}{\Delta \varepsilon} . \quad n=1,2, \cdots
$$

Since $\Delta \varepsilon>0$, the previous equation only has solution for $\delta>0$. The condition (32) is satisfied by the limit of weak coupling between matter and electric field $\left(D_{0} \ll \frac{\Delta \varepsilon}{2}\right)$. On the other hand, for $n \geq 2$, the equality (31) can not be accomplished by a weak coupling. 
2) $\Gamma=m \omega, \quad m=\frac{1}{2}, \frac{3}{2}, \frac{5}{2}, \cdots$

In this case, any vector state (14), with $c_{1}(0)$ and $c_{2}(0) \in \mathbb{C}$, acquires at $t=T$ the global phase

$$
\phi=-\frac{1}{2}\left(\varepsilon_{1}+\varepsilon_{2}\right) T .
$$

Subtracting the contribution of the dynamical phase from the global phase (33), we obtain the Aharonov-Anandan phase

$$
\begin{aligned}
& \beta=-\frac{\pi}{\cos (\theta)}\left(\frac{\Delta \varepsilon}{\omega}\right)\left[\left(\left|c_{2}(0)\right|^{2}-\left|c_{1}(0)\right|^{2}\right)\left(1-\frac{\sin ^{2}(\theta)}{4 m^{2}}\right)\right. \\
& \left.+\frac{\sin (\theta)}{2 m} \sqrt{1-\frac{\sin ^{2}(\theta)}{4 m^{2}}}\left|c_{1}(0)\right|\left(c_{2}(0) e^{-i \delta_{1}}+c_{2}^{*}(0) e^{i \delta_{1}}\right)\right],
\end{aligned}
$$

with $m=\frac{1}{2}, \frac{3}{2}, \frac{5}{2}, \cdots$

Again we verify that the $\beta$ phase depends on the initial vector state. We remind that $c_{1}(0)=\left|c_{1}(0)\right| e^{i \delta_{1}}$, with $\delta_{1} \in \mathbb{R}$. Comparing the phases (29) and (34), we see that they have similar expressions up to a $\pi$-phase.

To satisfy the condition $\Gamma=m \omega, m=\frac{1}{2}, \frac{3}{2}, \frac{5}{2}, \cdots$, in the quasi-resonant regime, the coupling $D_{0}$ has to be

$$
\left(\frac{D_{0}}{\Delta \varepsilon}\right)^{2} \approx\left(m^{2}-1\right)+\left(2 m^{2}-1\right)\left(\frac{\delta}{\Delta \varepsilon}\right), \quad m=\frac{1}{2}, \frac{3}{2}, \frac{5}{2}, \cdots
$$

We verify that eq.(35) does not have solution for $m=\frac{1}{2}$. For $m=\frac{3}{2}, \frac{5}{2}, \cdots$, we see again that the condition (35) can not be fulfilled by a weak coupling between the matter and the electric field.

We now consider a third condition among the frequencies $\Gamma$ and $\omega$, which were not previously discussed in Refs. [13, 14].

3) $\Gamma \neq l \omega, \quad l=\frac{1}{2}, 1, \frac{3}{2}, 2 \cdots$

For the vector state to satisfy the cyclic condition (20) at $\tau=T$, the initial coefficients $c_{1}(0)$ and $c_{2}(0)$ has to satisfy the coupled linear equations,

$$
\mathbf{M}\left(\begin{array}{l}
c_{1}(0) \\
c_{2}(0)
\end{array}\right)=\left(\begin{array}{l}
0 \\
0
\end{array}\right)
$$


The entries of the matrix $\mathbf{M}$ are,

$$
\begin{aligned}
& M_{11}=\Gamma \tilde{E}_{2}\left(\cos (\Gamma T)+e^{i \gamma}\right)+i \sin (\Gamma T)\left(\tilde{E}_{2}^{2}+\frac{\omega \Delta \varepsilon}{4}\right), \\
& M_{12}=M_{21}=-i \frac{\omega D_{0}}{2} \sin (\Gamma T), \\
& M_{22}=\Gamma \tilde{E}_{2}\left(\cos (\Gamma T)+e^{i \gamma}\right)-i \sin (\Gamma T)\left(\tilde{E}_{2}^{2}+\frac{\omega \Delta \varepsilon}{4}\right) .
\end{aligned}
$$

The phase $\gamma$ is defined as

$$
\gamma \equiv \phi+\frac{\left(\varepsilon_{1}+\varepsilon_{2}\right)}{2} T
$$

Eq.(36) has non-trivial solution only if that

$$
\operatorname{det}(\mathbf{M})=0 .
$$

From the entries of matrix $\mathbf{M}$, we obtain

$$
\operatorname{det}(\mathbf{M})=\Gamma^{2} \tilde{E}_{2}^{2}\left(1+2 \cos (\Gamma T) e^{i \gamma}+e^{2 i \gamma}\right) .
$$

The r.h.s. of the eq.(40) satisfies condition (39) when

$$
\gamma=\pi \pm \Gamma T
$$

and consequently we obtain the global phase

$$
\phi=\pi \pm-\frac{\left(\varepsilon_{1}+\varepsilon_{2}\right) T}{2} .
$$

Solving eq.(36) at $\phi=\pi \pm-\frac{\left(\varepsilon_{1}+\varepsilon_{2}\right) T}{2}$ and imposing that the coefficients $c_{1}(0)$ and $c_{2}(0)$ satisfy the normalization condition, we obtain

$$
\begin{aligned}
& c_{1}(0)=\frac{\omega D_{0} e^{i \delta_{1}}}{\left[\omega^{2} D_{0}^{2}+4\left[\tilde{E}_{2}\left(\tilde{E}_{2} \mp \Gamma\right)+\frac{\omega \Delta \varepsilon}{4}\right]^{2}\right]^{\frac{1}{2}}}, \\
& c_{2}(0)=\frac{2\left[\tilde{E}_{2}\left(\tilde{E}_{2} \mp \Gamma\right)+\frac{\omega \Delta \varepsilon}{4}\right] e^{i \delta_{1}}}{\left[\omega^{2} D_{0}^{2}+4\left[\tilde{E}_{2}\left(\tilde{E}_{2} \mp \Gamma\right)+\frac{\omega \Delta \varepsilon}{4}\right]^{2}\right]^{\frac{1}{2}}},
\end{aligned}
$$


with $\delta_{1} \in \mathbb{R}$. From the previous equations, we verify that only for particular values of the coefficients $c_{1}(0)$ and $c_{2}(0)$ the vector state satisfy the condition (20) at $\tau=T$ when $\Gamma \neq l \omega, l=\frac{1}{2}, 1, \frac{3}{2}, 2 \cdots$

The Aharonov-Anandan phase acquired by the vector state at $\tau=T$ when $\phi=$ $-\frac{\left(\varepsilon_{1}+\varepsilon_{2}\right) T}{2}+\pi \pm \Gamma T$ is

$$
\begin{aligned}
\beta & =\pi \pm \Gamma T-\frac{\pi}{\cos (\theta)}\left(\frac{\Delta \varepsilon}{\omega}\right)\left\{\left(\left|c_{2}(0)\right|^{2}-\left|c_{1}(0)\right|^{2}\right)\left[1-\frac{\omega^{2}}{4 \Gamma^{2}} \sin ^{2}(\theta)\left(1-\frac{\omega}{4 \pi \Gamma} \sin (2 \Gamma T)\right)\right]\right. \\
& \left.+\frac{\omega}{\Gamma}\left(\frac{1}{2}-\frac{\omega}{8 \pi \Gamma} \sin (2 \Gamma T)\right) \sin (\theta) \sqrt{1-\frac{\omega^{2}}{4 \Gamma^{2}} \sin ^{2}(\theta)}\left|c_{1}(0)\right|\left(c_{2}(0) e^{-i \delta_{1}}+c_{2}^{*}(0) e^{i \delta_{1}}\right)\right\} .
\end{aligned}
$$

The coefficients $c_{1}(0)$ and $c_{2}(0)$ in the previous equation are given by expression (43a) and (43b) respectively. In this case, the $\beta$ phase is independent of the value of $\delta_{1} \in \mathbb{R}$.

As a final comment on the cyclic evolution of the vector state during the period of the external electric field, we should notice that if the ratio

$$
\frac{\Gamma}{\omega}=\frac{m}{2 n}, \quad m, n=1,2,3, \cdots
$$

then at $\tau=n T$, the vector state $|\psi(n T)\rangle$ satisfies the condition (20) for any initial vector state, and the global $\phi$ phase is

$$
\phi=-\frac{1}{2}\left(\varepsilon_{1}+\varepsilon_{2}\right) n T+(m-n) T
$$

with $m, n=1,2,3, \cdots$.

\subsection{Cyclic evolution at $\tau \neq n T, n=1,2,3, \cdots$}

When the cyclic condition (20) is verified at $\tau \neq n T, n=\frac{1}{2}, 1, \frac{3}{2}, 2, \cdots$, we have $\left|\phi_{j} ; \tau\right\rangle \neq$ $\left|\phi_{j} ; 0\right\rangle, j=1,2$. Moreover, we note that for the $\tau \neq n T, n=1,2,3, \cdots$, the classical electric field does not travel a closed path in its parameter space when the initial state vector acquires a phase.

In order the discuss the condition (20) at $\tau \neq n T$, it is better to write the states $|\psi(0)\rangle$ and $|\psi(\tau)\rangle$ in the basis of the eigenstates of hamiltonian $\mathbf{H}_{0}$. To fulfill the condition (20), the initial coefficients $c_{1}(0)$ and $c_{2}(0)$ in the initial vector state (14) have to satisfy the coupled linear equations:

$$
\tilde{\mathbf{M}}\left(\begin{array}{l}
c_{1}(0) \\
c_{2}(0)
\end{array}\right)=\left(\begin{array}{l}
0 \\
0
\end{array}\right)
$$


The entries of the matrix $\tilde{\mathbf{M}}$ are

$$
\begin{aligned}
& \tilde{M}_{11}=\sin \left(\frac{\theta}{2}\right)\left[e^{i \tilde{\gamma}}-\cos (\Gamma \tau)\right]+i \frac{\sin (\Gamma \tau)}{\Gamma}\left[-\sin \left(\frac{\theta}{2}\right)\left(\tilde{E}_{2}-\frac{\omega \cos (\theta)}{2}\right)-\frac{\omega \sin (\theta)}{2} \cos \left(\frac{\theta}{2}\right)\right], \\
& \tilde{M}_{12}=-\cos \left(\frac{\theta}{2}\right)\left[e^{i \tilde{\gamma}}-\cos (\Gamma \tau)\right]-i \frac{\sin (\Gamma \tau)}{\Gamma}\left[\cos \left(\frac{\theta}{2}\right)\left(\tilde{E}_{2}-\frac{\omega \cos (\theta)}{2}\right)-\frac{\omega \sin (\theta)}{2} \sin \left(\frac{\theta}{2}\right)\right], \\
& \tilde{M}_{21}=\cos \left(\frac{\theta}{2}\right)\left[-e^{i(\tilde{\gamma}-\omega \tau)}+\cos (\Gamma \tau)\right]+i \frac{\sin (\Gamma \tau)}{\Gamma}\left[\cos \left(\frac{\theta}{2}\right)\left(\tilde{E}_{2}-\frac{\omega \cos (\theta)}{2}\right)-\frac{\omega \sin (\theta)}{2} \sin \left(\frac{\theta}{2}\right)\right], \\
& \tilde{M}_{22}=\sin \left(\frac{\theta}{2}\right)\left[-e^{i(\tilde{\gamma}-\omega \tau)}+\cos (\Gamma \tau)\right]+i \frac{\sin (\Gamma \tau)}{\Gamma}\left[-\sin \left(\frac{\theta}{2}\right)\left(\tilde{E}_{2}-\frac{\omega \cos (\theta)}{2}\right)-\frac{\omega \sin (\theta)}{2} \cos \left(\frac{\theta}{2}\right)\right] .
\end{aligned}
$$

The phase $\tilde{\gamma}$ is defined as

$$
\tilde{\gamma}(\tau) \equiv \phi+\frac{\left(\varepsilon_{1}+\varepsilon_{2}+\omega\right) \tau}{2}
$$

The coupled eqs.(47) have non-trivial solution only if $\operatorname{det}(\tilde{\mathbf{M}})=0$. By direct calculation we obtain

$$
\operatorname{det}(\tilde{\mathbf{M}})=-1+\left[\cos (\Gamma \tau)\left(e^{i \tilde{\gamma}}+e^{i(\tilde{\gamma}-\omega \tau)}\right)-e^{i(2 \tilde{\gamma}-\omega \tau)}\right]-i \frac{\sin (\Gamma \tau)}{2 \Gamma}(\omega+\Delta \varepsilon)\left[e^{i \tilde{\gamma}}-e^{i(\tilde{\gamma}-\omega \tau)}\right]
$$

All the cases discussed in subsection 3.1 satisfy identically the condition $\operatorname{det}(\tilde{\mathbf{M}})=0$ when the relations between $\Gamma$ and $\omega$ are substituted on the r.h.s. of eq.(50).

We will not discuss the general case when (50) is null for the arbitrary value of $\tau$.

Up to the end of this subsection, we take $\tau=T_{\Gamma}, T_{\Gamma}$ being the period associated to the Rabi's frequency.

The vector state at $\tau=T_{\Gamma}$ is

$$
\begin{array}{r}
\left|\psi\left(T_{\Gamma}\right)\right\rangle=e^{-\frac{i}{2}\left(\varepsilon_{1}+\varepsilon_{2}+\omega\right) T_{\Gamma}}\left\{\left[-\sin \left(\frac{\theta}{2}\right) c_{1}(0)+\cos \left(\frac{\theta}{2}\right) c_{2}(0)\right]|1\rangle\right. \\
\left.+e^{-i \phi_{0}} e^{i \omega T_{\Gamma}}\left[\cos \left(\frac{\theta}{2}\right) c_{1}(0)+\sin \left(\frac{\theta}{2}\right) c_{2}(0)\right]|2\rangle\right\} .
\end{array}
$$


The vector state (51) is equal to the initial state (14) up to a global phase, independently of the values of $c_{1}(0)$ and $c_{2}(0)$, if

$$
\Gamma=\frac{\omega}{n}, \quad n=1,2,3, \cdots
$$

The previous relation is identical to $T_{\Gamma}=n T$.

In the quasi-resonant regime, eq.(52) implies

$$
\left(\frac{D_{0}}{\Delta \varepsilon}\right)^{2} \approx\left(\frac{1}{n^{2}}-1\right)+\left(\frac{2}{n^{2}}-1\right)\left(\frac{\delta}{\Delta \varepsilon}\right), \quad n=1,2,3, \cdots
$$

Eq.(53) has solution only for $n=1$ when $\delta>0$.

The global phase $\phi$ acquired by any vector state at $\tau=T_{\Gamma}$, for $n=1$ in the condition $(52)$ is

$$
\phi=-\frac{1}{2}\left(\varepsilon_{1}+\varepsilon_{2}\right) T_{\Gamma}-\pi
$$

The Aharonov-Anandan phase in this case is

$$
\begin{aligned}
\beta=-\pi & -\frac{\pi}{\cos (\theta)}\left(\frac{\Delta \varepsilon}{\omega}\right)\left[\left(\left|c_{2}(0)\right|^{2}-\left|c_{1}(0)\right|^{2}\right)\left(1-\frac{\sin ^{2}(\theta)}{4}\right)\right. \\
& \left.+\frac{\sin (\theta)}{2} \sqrt{1-\frac{\sin ^{2}(\theta)}{4}}\left|c_{1}(0)\right|\left(c_{2}(0) e^{-i \delta_{1}}+c_{2}^{*}(0) e^{i \delta_{1}}\right)\right] .
\end{aligned}
$$

This $\beta$ phase also depends on the initial vector state.

As our final case we continue to have $\tau=T_{\Gamma}$ but $T_{\Gamma} \neq n T$, with $n=1,2,3, \cdots$

The vector state $\left|\psi\left(T_{\Gamma}\right)\right\rangle$ continues to be given by expression (51). Since $T_{\Gamma} \neq n T$, $n=1,2, \cdots$, we verify that is not possible for any coefficients $c_{1}(0)$ and $c_{2}(0) \in \mathbb{C}$ that $\mid \psi\left(T_{\Gamma}\right\rangle$ satisfy the condition (20).

At $\tau=T_{\Gamma}$, we decompose the $\operatorname{det}(\tilde{M})$ in its real and imaginary parts,

$$
\begin{aligned}
\operatorname{det}(\tilde{M})=2 \sin \left(\frac{\gamma^{\prime}}{2}\right) & \left\{\left[-\sin \left(\frac{\gamma^{\prime}}{2}\right)+\sin \left(\frac{3 \gamma^{\prime}}{2}-\omega T_{\Gamma}\right)\right]\right. \\
+ & \left.2 i \sin \left(\frac{\gamma^{\prime}-\omega T_{\Gamma}}{2}\right) \sin \left(\gamma^{\prime}-\frac{\omega T_{\Gamma}}{2}\right)\right\},
\end{aligned}
$$

where $\gamma^{\prime}=\tilde{\gamma}\left(T_{\Gamma}\right)$.

To have $\operatorname{det}(\tilde{M})=0$, the real and imaginary parts on the r.h.s. of (파) have to be simultaneously null. For arbitrary value of $\omega T_{\Gamma}$, one solution to $\operatorname{det}(\tilde{M})=0$ is

$$
\gamma^{\prime} \sim 0, \quad \bmod (2 \pi)
$$


To calculate the values of the initial coefficients $c_{1}(0)$ and $c_{2}(0)$ such that the vector $\left|\psi\left(T_{\Gamma}\right)\right\rangle$ acquires the global phase

$$
\phi=-\frac{\left(\varepsilon_{1}+\varepsilon_{2}\right) T_{\Gamma}}{2}-\frac{\omega T_{\Gamma}}{2}
$$

and the Aharonov-Anandan phase

$$
\begin{aligned}
\beta & =-\frac{\pi \omega}{\Gamma}-\frac{\pi}{\cos (\theta)}\left(\frac{\omega}{\Gamma}\right)\left(\frac{\Delta \varepsilon}{\omega}\right)\left\{\left[\left|c_{2}(0)\right|^{2}-\left|c_{1}(0)\right|^{2}\right]\left(1-\frac{\omega^{2}}{4 \Gamma^{2}} \sin ^{2}(\theta)\right)\right. \\
& \left.+\frac{\omega}{2 \Gamma} \sin (\theta) \sqrt{1-\frac{\omega^{2}}{4 \Gamma^{2}} \sin ^{2}(\theta)}\left|c_{1}(0)\right|\left[c_{2}(0) e^{-i \delta_{1}}+c_{2}^{*}(0) e^{i \delta_{1}}\right]\right\}
\end{aligned}
$$

the solution (57) has to be replaced on the coupled eq.(47).

Besides the solution (57), the real part of the r.h.s. of (56) has the common root:

$$
\gamma^{\prime} \sim \omega T_{\Gamma}, \quad \bmod (2 \pi)
$$

valid for arbitrary value of $\omega T_{\Gamma}$.

The vector state at $\tau=T_{\Gamma}$, for particular values of $c_{1}(0)$ and $c_{2}(0)$, acquires the global phase

$$
\phi \sim-\frac{1}{2}\left(\varepsilon_{1}+\varepsilon_{2}\right) T_{\Gamma}+\frac{\omega T_{\Gamma}}{2}, \quad \bmod (2 \pi)
$$

The Aharonov-Anandan phase (the $\beta$ phase) in this case is equal to

$$
\begin{aligned}
\beta & =\frac{\pi \omega}{\Gamma}-\frac{\pi}{\cos (\theta)}\left(\frac{\omega}{\Gamma}\right)\left(\frac{\Delta \varepsilon}{\omega}\right)\left\{\left[\left|c_{2}(0)\right|^{2}-\left|c_{1}(0)\right|^{2}\right]\left(1-\frac{\omega^{2}}{4 \Gamma^{2}} \sin ^{2}(\theta)\right)\right. \\
& \left.+\frac{\omega}{2 \Gamma} \sin (\theta) \sqrt{1-\frac{\omega^{2}}{4 \Gamma^{2}} \sin ^{2}(\theta)}\left|c_{1}(0)\right|\left[c_{2}(0) e^{-i \delta_{1}}+c_{2}^{*}(0) e^{i \delta_{1}}\right]\right\}
\end{aligned}
$$

\section{Conclusions}

We study the dynamical evolution of a two-level model coupled to a classical linearly polarized monochromatic electric field in the RWA. We map this model onto a spin- $1 / 2$ model coupled to an effective magnetic field. The dynamics of the latter model has already been studied in the literature [17, 18, for any regime. In particular, its Aharonov-Anandan phases were studied in Refs. [13, 14] to the condition $\Gamma=n \omega, n=\frac{1}{2}, 1, \frac{3}{2}, 2, \cdots$

Our results on the dynamics of the RWA of the electric two-level model (see hamiltonian (21) ) are valid for any value of the ratio $\frac{\omega}{\Delta \varepsilon}$. In particular, this model in the 
quasi-resonant regime is a good description of the coupling between matter and an electric field in the electric dipole approximation, being a candidate for modeling a gate in quantum computation[2, 7].

We show that when a cycle of the vector state occurs within a cycle of the external electric field, the Aharonov-Anandan phase depends on the initial vector state, unless the quantum system evolves adiabatically. Our results are in disagreement with Refs. [8, 9,

We also show that the periodic behavior of the vector state (see eq.(28)) can also happen within the period $T_{\Gamma}$ associated to Rabi's frequency (19b). Again, the AharonovAnandan phase depends on the initial coefficients of the initial vector state.

In both cases, we verified that in the non-adiabatic regime the Aharonov-Anandan phase depends on the initial state and on the interval of time for the vector state to return to its initial state, up to a global phase. Although our results are valid for any value of the $\frac{\omega}{\Delta \varepsilon}$, part of our results are discussed in the quasi-resonant regime, where we expect our results to be applicable to the modeling of a quantum computation device.

Our results are easily reinterpreted in terms of the spin-1/2 model in the presence of a rotating magnetic field. They extend previous discussions [12, 13, 14] on the AharonovAnandan phases of this model.

The authors thank E.V. Corrêa Silva for the careful reading of part of this manuscript. M.T.T. is in debt with S.A. Dias for the nice discussions on Ref.[11]. M.T. Thomaz (Fellowship CNPq, Brazil, Proc.No.: 30.0549/83-FA) thanks CNPq for partial financial support.

\section{Appendix A The average energy in any time- dependent vector state}

Ref.[11] writes the periodic condition of the vector state $|\psi(t)\rangle$ at $t=\tau$ as

$$
\begin{aligned}
|\psi(\tau)\rangle & =e^{i \phi}|\psi(0)\rangle \\
& =e^{i \beta} e^{-i \int_{0}^{\tau}\left\langle\psi(t)\left|\mathbf{H}_{e}(t)\right| \psi(t)\right\rangle d t}|\psi(0)\rangle,
\end{aligned}
$$

where the dynamical phase is diminished from the global phase $\phi$.

We assume the initial vector state (14) and let $c_{1}(0)$ and $c_{2}(0) \in \mathbb{C}$. The coefficient $c_{1}(0)$ is written as

$$
c_{1}(0)=\left|c_{1}(0)\right| e^{i \delta_{1}}
$$

with $\delta_{1} \in \mathbb{R}$.

The expectation value of the energy in the vector state (16), at any instant $t$, is 


$$
\begin{aligned}
\left\langle\psi(t)\left|\mathbf{H}_{e}(t)\right| \psi(t)\right\rangle & =\frac{\left(\varepsilon_{1}+\varepsilon_{2}\right)}{2}+\tilde{E}_{2}\left\{\left[\left|c_{2}(0)\right|^{2}-\left|c_{1}(0)\right|^{2}\right]\right. \\
& +\frac{i}{2} \frac{\omega}{\Gamma} \sin (\theta)\left|c_{1}(0)\right| \sin (2 \Gamma t)\left[c_{2}(0) e^{-i \delta_{1}}-c_{2}^{*}(0) e^{i \delta_{1}}\right] \\
+\sin ^{2}(\Gamma t)\left[-\frac{\omega^{2}}{2 \Gamma^{2}}\left[\left|c_{2}(0)\right|^{2}-\left|c_{1}(0)\right|^{2}\right] \sin ^{2}(\theta)\right. & \\
& \left.\left.+\frac{\omega}{\Gamma} \sin (\theta) \sqrt{1-\frac{\omega^{2}}{4 \Gamma^{2}} \sin ^{2}(\theta)}\left|c_{1}(0)\right|\left[c_{2}(0) e^{-i \delta_{1}}+c_{2}^{*}(0) e^{i \delta_{1}}\right]\right]\right\} .
\end{aligned}
$$

\section{References}

[1] P. Meystre and Murray Sargent III, "Elements of Quantum Optics", $3^{\text {rd }}$ ed., SpringerVerlag (1998), section 3.3.

[2] M. A. Nielsen and I. L. Chuang, "Quantum Computation and Quantum Information", Cambridge Univ. Press (2007), chap. 7.

[3] I.I. Rabi, Phys. Rev. 49, 324-328 (1936).

[4] I.I. Rabi, Phys. Rev. 51, 652-654 (1937).

[5] A.C. Aguiar Pinto, M. Moutinho and M.T. Thomaz, Braz. J. of Phys. 39, 326-330 (2009).

[6] J.C. Garrison and E.M. Wright, Phys. Lett. A 128, 177-181 (1988).

[7] X.-Q. Li et al., Phys. Rev. A 66, 042320 (2002).

[8] H. Imai, Y. Otsubo and A. Morinaga, Phys. Rev. A 76, 012116-1-4 (2007).

[9] H. Imai and A. Morinaga, Phys. Rev. A 76, 062111-1-4 (2007).

[10] M. V. Berry, Proc. R. Soc. London, Ser. A 392, 45-57 (1984).

[11] Y. Aharonov and J. Anandan, Phys. Rev. Lett. 58, 1593-1596 (1987).

[12] A. Bulgac, Phys. Rev. A 37, 4084 (1988).

[13] G.-j. Ni, S.-q. Chen and Y.-l. Shen, Phys. Lett A 197, 100 (1995).

[14] S.-L. Zhu, Z.D. Wang and Y.-D. Zhang, Phys. Rev. B 61, 1142 (2000). 
[15] C. Cohen-Tannoudji, B. Diu and F. Laloë, "Quantum Mechanics", vol.1, John Wiley \& Sons (1977, Paris), page 405.

[16] W. E. Lamb Jr., R.R. Schicher and M.O. Scully, Phys. Rev. A 36, 2763-2772 (1987).

[17] A.C. Aguiar Pinto, M.C. Nemes, J.G. Peixoto de Faria and M.T. Thomaz, Am. J. Phys. 68, 955-958 (2000).

[18] G. García de Polavieja and E. Sjöqvist, Am. J. Phys. 66, 431-438 (1998) and references therein. 